\title{
Microstructure and Segmental Dynamics of Polyurea under Uniaxial Deformation
}

\author{
Taeyi Choi ${ }^{\dagger}$ Daniel Fragiadakis, ${ }^{\dagger}$ C. Michael Roland, ${ }^{*} \stackrel{+}{\ddagger}$ and James Runt ${ }^{*} \dagger$ \\ ${ }^{\dagger}$ Department of Materials Science and Engineering, The Pennsylvania State University, University Park, Pennsylvania 16802, \\ United States \\ ${ }^{\ddagger}$ Chemistry Division, Naval Research Laboratory, Code 6120, Washington, D.C. 20375, United States
}

ABSTRACT: Polyureas, formed by the rapid reaction between isocyanates and diamines, are attractive for various applications due to their outstanding mechanical properties, which can be tuned by varying component chemistry, molecular weight, and stoichiometry. Polyureas synthesized from a modified methylene diphenyl diisocyanate (Isonate $143 \mathrm{~L}$ ) and polytetramethylene oxide-di-p-aminobenzoate (Versalink P1000) are widely utilized and investigated for energy absorbing applications such as impact mitigation and ballistic protection. In order to develop a more complete understanding of their mechanical response, we explore the effect of uniaxial strain on the phase separated microstructure and molecular dynamics. We utilize wide- and small-angle X-ray scattering to investigate amorphous segment and hard domain orientation, and broadband dielectric spectroscopy for interrogation of the dynamics. Uniaxial deformation was found to significantly perturb the phase-separated microstructure and chain orientation and result in a considerable slowing down and broadening of the polyurea soft phase segmental relaxation.

\section{INTRODUCTION}

Polyureas (PU) are formed from the reaction of isocyanates, usually based on diphenylmethane diisocyanate (MDI), with polyetheramines. The reaction is rapid with gel times measured in minutes, which means the reaction proceeds largely independently of ambient temperature and humidity, facilitating application of PU under diverse conditions. Polyureas have been used commercially since the early 1990s, with their processing and properties leading to widespread applications. In particular, the extensive hydrogen-bonding of PU leads to exceptional mechanical "toughness". Applications for PU include concrete coatings, repair of roofs and parking decks, and linears for storage tanks, freight ships, and truck beds. Prominent examples include the Boston tunnel project, the Incheon, Korea, airport, and the San Mateo, CA, bridge. PU coatings have also been found to mitigate the effects of bomb blasts on buildings by suppressing fragmentation, and thus have been applied to the Pentagon and various American embassies. A related application is the use of PU coatings as a means to up-armor military vehicles, such as the Humvee.

Reflecting the performance advantages of the material and the desire to further its application, there has been a large amount of experimental research lately aimed at better understanding the structure, ${ }^{1}$ molecular dynamics, ${ }^{2,3}$ mechanical properties ${ }^{4-8}$ (especially at high strain rates), and performance as a coating for improving blast and impact resistance, ${ }^{9-14}$ as well as efforts to create constitutive models ${ }^{15,16}$ and sophisticated multiscale models ${ }^{17}$ that predict the observed behaviors. The phaseseparated microstructure of polyurea is crucial in determining molecular dynamics and mechanical properties, and its evolution

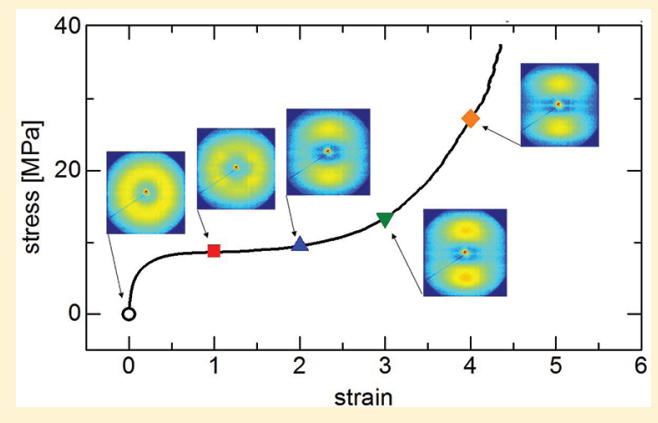

during mechanical loading and unloading was recently studied by Rinaldi et al. ${ }^{18}$ up to moderate strain.

The purpose of the present work was to determine how the structure of PU, its orientation, and the interaction of the phase domains, are affected by mechanical deformation. We employ $\mathrm{X}$-ray scattering and dielectric relaxation spectroscopy to probe the changes in morphology and segmental dynamics brought about by extensional straining through the yield point up to failure. The polyurea selected for the study is used as an acoustic material on submarines, and is being explored as a protective barrier against impact and ballistic assault. We quantify the changes in structure and dynamics transpiring during deformation and their relationship to the strain history.

\section{EXPERIMENTAL SECTION}

The polyurea was formed by reaction of poly(tetramethylene oxidedi-p-aminobenzoate) (Versalink P1000, Air Products) and a polycarbodiimidemodified diphenylmethane diisocyanate (Isonate $143 \mathrm{~L}$, Dow Chemical) at a 4:1 weight ratio. The molecular weight given by the manufacturer for the PTMO-amine component is $M=1238 \pm$ $72 \mathrm{~g} / \mathrm{mol}$. The components were degassed under vacuum, mixed at $40{ }^{\circ} \mathrm{C}$, and poured into a Teflon mold. Reaction was carried out at room temperature for $8 \mathrm{~h}$, followed by an additional $12 \mathrm{~h}$ at $353 \mathrm{~K}$.

Stress-strain measurements were carried out on an Instron 5500R with video extensometer; the nominal strain rate was $0.2 \mathrm{~s}^{-1}$. Separate samples were held at $100 \%, 200 \%, 300 \%$, and $400 \%$ strain until the

Received: January 18, 2012

Revised: $\quad$ March 26, 2012

Published: April 6, 2012 
stress became constant, then clamped into aluminum frames and used for the X-ray scattering and dielectric spectroscopy experiments.

To investigate segment and hard domain orientations during uniaxial deformation, $\mathrm{X}$-ray scattering experiments were conducted at two different length scales. To follow overall segment orientation, wide-angle X-ray diffraction (WAXD) patterns were measured on a Rigaku DMAX/rapid microdiffractometer in transmission mode, using a copper point-focused source $(\lambda=0.154 \mathrm{~nm})$ at $50 \mathrm{kV}$ and $40 \mathrm{~mA}$. Small-angle X-ray scattering (SAXS) experiments were used to explore hard domain orientation and any changes in unlike segment mixing. The SAXS data were collected using a Molecular Metrology instrument with a $\mathrm{Cu} \mathrm{K} \alpha$ radiation source $(\lambda=0.154 \mathrm{~nm})$ at $45 \mathrm{kV}$ and $66 \mathrm{~mA}$, using a two-dimensional multiwire detector with a sample-to-detector distance of $1.5 \mathrm{~m}$. Absolute scattered intensities were determined by comparing the spectra of the samples to that of a precalibrated, cross-linked polyethylene (S-2907) secondary standard. ${ }^{19}$ SAXS and WAXD measurements were also conducted after specimens were relaxed for $24 \mathrm{~h}$ at ambient conditions in order to evaluate the extent of recovery after particular tensile strains.

Dielectric spectroscopy measurements were carried out using a parallel plate geometry with the sample in the form of a disk (15-20 $\mathrm{mm}$ diameter, $0.2-0.3 \mathrm{~mm}$ thick). Spectra were obtained as a function of temperature using a Novocontrol Alpha analyzer $\left(10^{-2}-10^{6} \mathrm{~Hz}\right)$. The temperature was controlled using a Delta Design model 9023 oven. Both dry and hydrated samples were measured. For the former, samples were placed in a vacuum oven at $40{ }^{\circ} \mathrm{C}$ for several days, then quickly transferred to the test chamber and measured under dry nitrogen. "Hydrated" samples were exposed to saturated water vapor for several days prior to measurement. The final water content was $2.4,2.6,2.7,2.9$, and $3.2 \%$ for samples having respective strains of $0,100,200,300$, and $400 \%$ strain. In order to maintain constant water content, the hydrated samples were submerged in a fluorinated silicone oil during the dielectric measurements. It was verified that the presence of the oil did not significantly affect the measured spectra.

\section{RESULTS AND DISCUSSION}

Stress-Strain Behavior. Figure 1 shows a representative engineering stress-strain curve for the polyurea under investigation. Beyond an initial linear region, the material yields, with a long plateau of nearly constant stress, followed by a large upturn in stress prior to failure. The inset shows the permanent set (unrecovered strain) as a function of applied strain. This set is about $10 \%$ of the strain, becoming significant at applied strains exceeding $400 \%$. The five points denoted in Figure 1 indicate

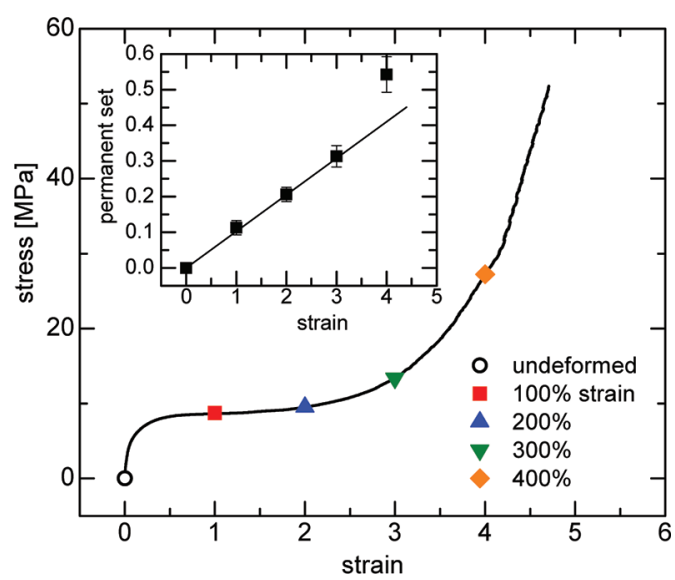

Figure 1. Representative stress-strain curve of polyurea under investigation. The points indicate the strains at which dielectric measurements were performed. Inset: permanent set (after relaxing to equilibrium) as a function of strain. the strains at which the structure and dynamics measurements were carried out; these correspond to the undeformed material, at the beginning and midpoint of the plateau, and at the initial and middle parts of the steep rise prior to failure, respectively.

Chain Orientation: Wide-Angle X-ray Diffraction. The undeformed polyurea (Figure 2) exhibits predominately isotropic scattering, with slightly stronger scattered intensity on the equator, giving rise to a broad amorphous halo in the azimuthally averaged one-dimensional WAXD profiles shown in Figure 3. As the extension ratio increases, the scattered intensity becomes concentrated on the equator, indicating that the (amorphous) segments are increasingly oriented along the deformation direction.

2D X-ray diffraction patterns after relaxation from various strains are displayed in Figure 2. (The background color and contrast of the top and bottom row of Figure 2 are different, so only differences between the equators and meridians should be compared.) After relaxation, diffraction patterns have less equatorial intensity, indicating recovery of the aligned segments, although samples exposed to high strains $(\geq 300 \%)$ exhibit some residual alignment and permanent deformation, consistent with the larger permanent set observed in Figure 1.

The corresponding azimuthally averaged scattered intensity profiles are displayed in Figure 3. Regardless of the extent of deformation, we only observe an amorphous halo at $2 \theta=20^{\circ}$ (4.4 $\AA$ ), arising from the mean spacing between species, demonstrating that hard segments do not organize into a $3 \mathrm{D}$ crystalline structure under the deformation conditions employed herein.

To quantitatively evaluate the degree of segment orientation, the Hermans' orientation function $\left(f_{x}\right)$ was calculated from the WAXD at angles between $2 \theta=18-22^{\circ}$ using $^{20,21}$

$$
\begin{aligned}
& f_{x}=\frac{3\left\langle\cos ^{2} \Phi\right\rangle-1}{2} \\
& \left\langle\cos ^{2} \Phi\right\rangle=\frac{\int_{0}^{90^{\circ}} I(\Phi) \sin \Phi \cos ^{2} \Phi \mathrm{d} \Phi}{\int_{0}^{90^{\circ}} I(\Phi) \sin \Phi \mathrm{d} \Phi}
\end{aligned}
$$

where $\Phi$ is the azimuthal angle (equal to zero at the meridian and $90^{\circ}$ at the equator). The function $f_{x}$ varies from zero to unity for isotropic and perfect segment orientation, respectively. Orientation functions were also calculated in the same fashion from SAXS data. Figure 4 displays $f_{x}$ vs uniaxial strain. With increasing strain (filled data points), $f_{x}$ values increase from zero to 0.25 at $400 \%$ strain. After relaxation (open data points), $f_{x}$ decreases significantly, for example, from 0.25 to 0.05 at $400 \%$ strain, demonstrating that most of the segment-level alignment is recovered after relaxation and that this phenomenon is dominated by soft segment relaxation.

Domain Orientation: Small-Angle X-ray Scattering. Two-dimensional SAXS patterns of deformed and relaxed samples (identical to those used in the WAXD experiments) at the various tensile strains are displayed in Figure 5. As expected, the undeformed material exhibits an isotropic scattering pattern. At $100 \%$ strain, a four-point intensity pattern along with two weaker reflections are observed, the scattering behavior arising due to shearing at the microdomain level, induced by the local torque exerted by the strained soft segments. ${ }^{21-25} \mathrm{~A}$ similar four-point pattern was observed in deformed polyurea by 

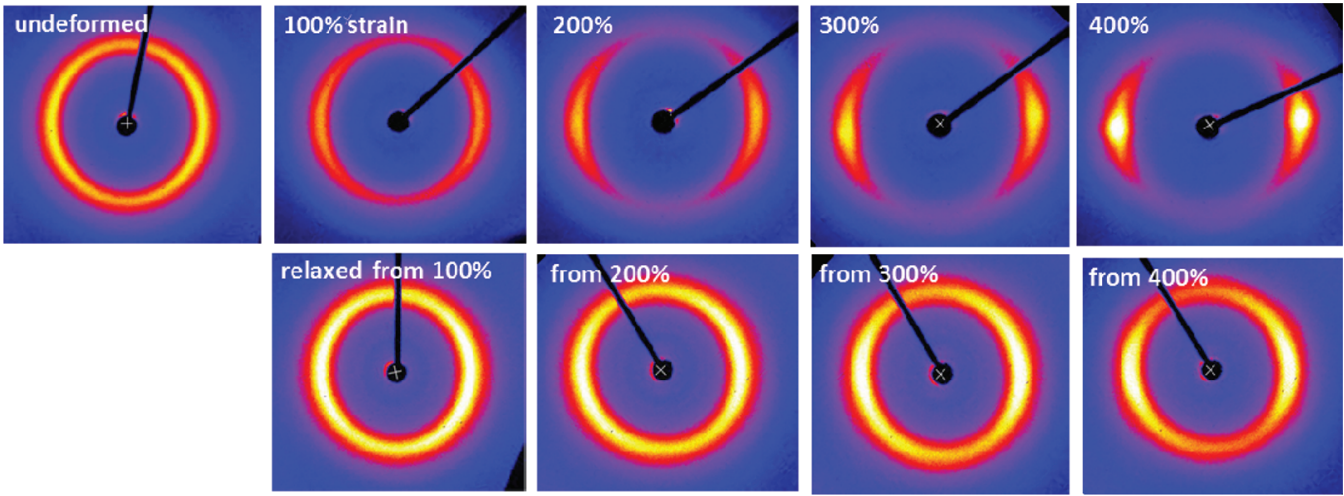

Figure 2. 2D WAXD patterns: (top row) undeformed polyurea and polyurea at $100 \%, 200 \%, 300 \%$ and $400 \%$ strain; (bottom row) polyureas after relaxation. The deformation direction is vertical.

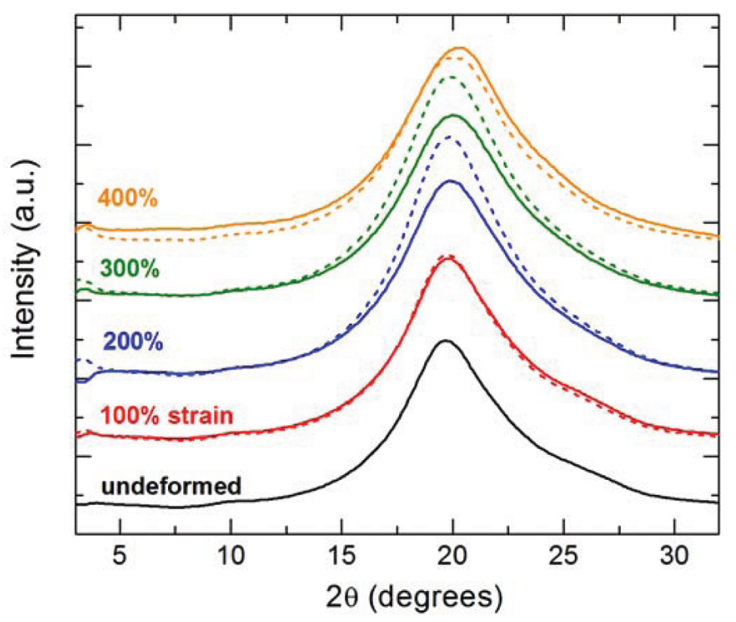

Figure 3. WAXD scattered intensity profiles as a function of $2 \theta$ (bottom to top): undeformed, $100 \%, 200 \%, 300 \%$, and $400 \%$ strain. Key: solid lines, deformed samples; dashed lines, samples after relaxation.

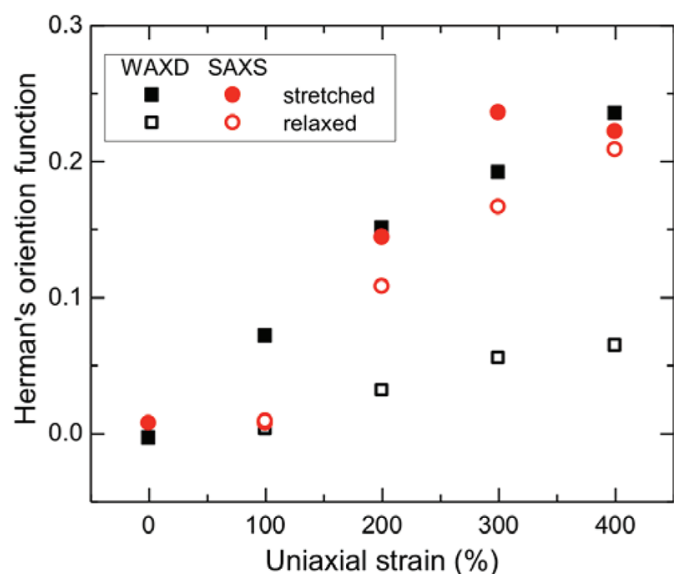

Figure 4. Hermans' orientation function for uniaxially deformed and relaxed polyureas: from WAXD measurements (squares) and from SAXS (circles).

Rinaldi et al. ${ }^{18}$ The four and two-point patterns are seen clearly in Figure 6, which displays the angular intensity dependence vs azimuthal angle. At $100 \%$ strain, we observe a four point pattern with maxima at $55^{\circ}, 125^{\circ}, 235^{\circ}$, and $305^{\circ}$, and less intense reflections at $90^{\circ}$ and $180^{\circ}$. At higher strains ( $\left.\geq 200 \%\right)$, a meridional two-point scattering pattern emerges, arising from hard domain reorganization and orientation due to stress transferred from oriented soft segments. ${ }^{21,25}$

In addition to the strong scattered intensity on the meridian, streaks appear perpendicular to the stretching direction at $400 \%$ strain. Such behavior has been observed previously and ascribed to formation of nanofibrils on deformation..$^{21,24-26}$

SAXS measurements were conducted on the identical samples after removing the tensile strain to characterize hard domain rearrangement. As displayed in Figure 5, for strains $\geq 200 \%$ significant scattering remains concentrated on the meridians after relaxation. The equatorial streaking for the $400 \%$ strained sample disappears after relaxation, illustrating that the nanofibrillar texture is not preserved on strain removal.

To quantify hard domain orientation, the Hermans' orientation factor from the SAXS data is plotted vs uniaxial strain in Figure 4. At strains $\geq 200 \%, f_{x}$ increases up to $\sim 0.2$ and remains essentially constant after relaxation, indicating that hard domains are oriented (plastically deformed) at high strains, with negligible relaxation after removal of the strain. This is distinct from the substantial recovery at larger scale of oriented segments, as reflected in the WAXD analysis; clearly the latter is dominated by soft segments. Finally, the calculated orientation factor for the $100 \%$ strained sample is complicated by the hard domain reorganization at $100 \%$ strain, as seen in Figures 5 and 6 , which is retained after relaxation.

Plots of azimuthally averaged absolute scattering intensities vs $q$ before and after deformation are displayed in Figure 7 . The mean interdomain spacing $\left(d=2 \pi / q_{\max }\right)$ and experimental electron density variances $\left(\overline{\Delta \eta^{2 \prime}}\right)$, which include the effects of intermixed segments and diffuse phase boundaries, are listed in Table 1 . The variance is determined from the background corrected data ${ }^{27}$ using

$$
\overline{\Delta \eta^{2}}=c Q=c \int\left\{I(q)-I_{b}(q)\right\} q^{2} \mathrm{~d} q
$$

where $Q$ is the invariant and the constant $c$ is

$$
c=\frac{1}{2 \pi^{2} i_{e} N_{a v}{ }^{2}}=1.76 \times 10^{-24} \mathrm{~mol}^{2} / \mathrm{cm}^{2}
$$

The symbol $i_{e}$ represents the Thompson's constant for the scattering from one electron $\left(7.94 \times 10^{-26} \mathrm{~cm}^{2}\right)$ and $N_{a v}$ is Avogadro's number.

In two-phase polyureas, the ratio of $\overline{\Delta \eta^{2 \prime}}$ to the theoretical electron density variance for the case of complete unlike segment demixing $\left(\overline{\Delta \eta^{2}} / \overline{\Delta \eta_{c}^{2}}\right)$ provides a measure of the 

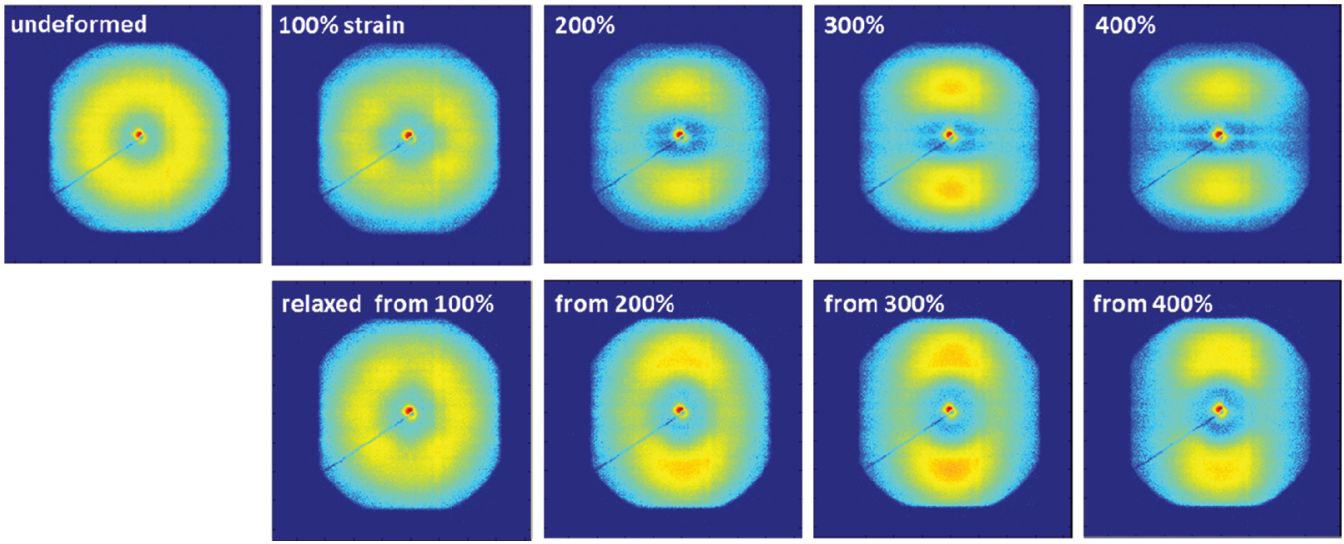

Figure 5. 2D SAXS patterns of polyurea: (top row) undeformed and deformed to various tensile strains; (bottom row) relaxed after deformation. Vertical deformation direction.
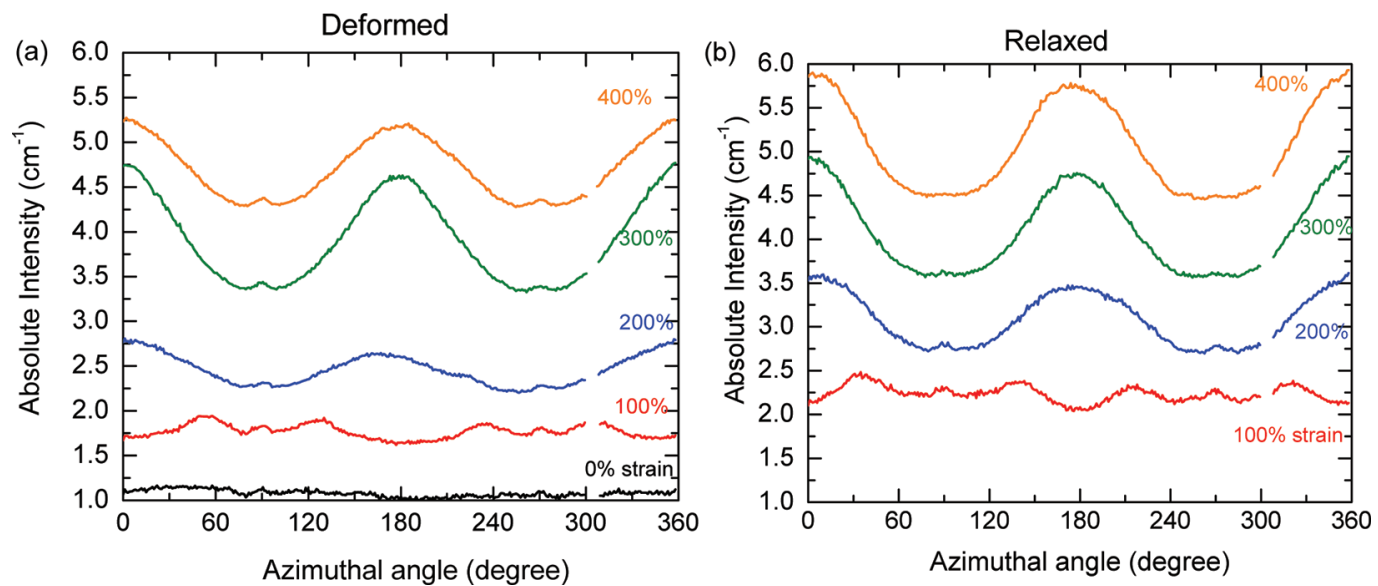

Figure 6. Background corrected absolute intensity plots vs azimuthal angle (a) under uniaxial deformation and (b) after relaxation. Curves have been shifted vertically for clarity by $1,2,3$, and $4 \mathrm{~cm}^{-1}$ for $100 \%, 200 \%, 300 \%$, and $400 \%$ strain, respectively.
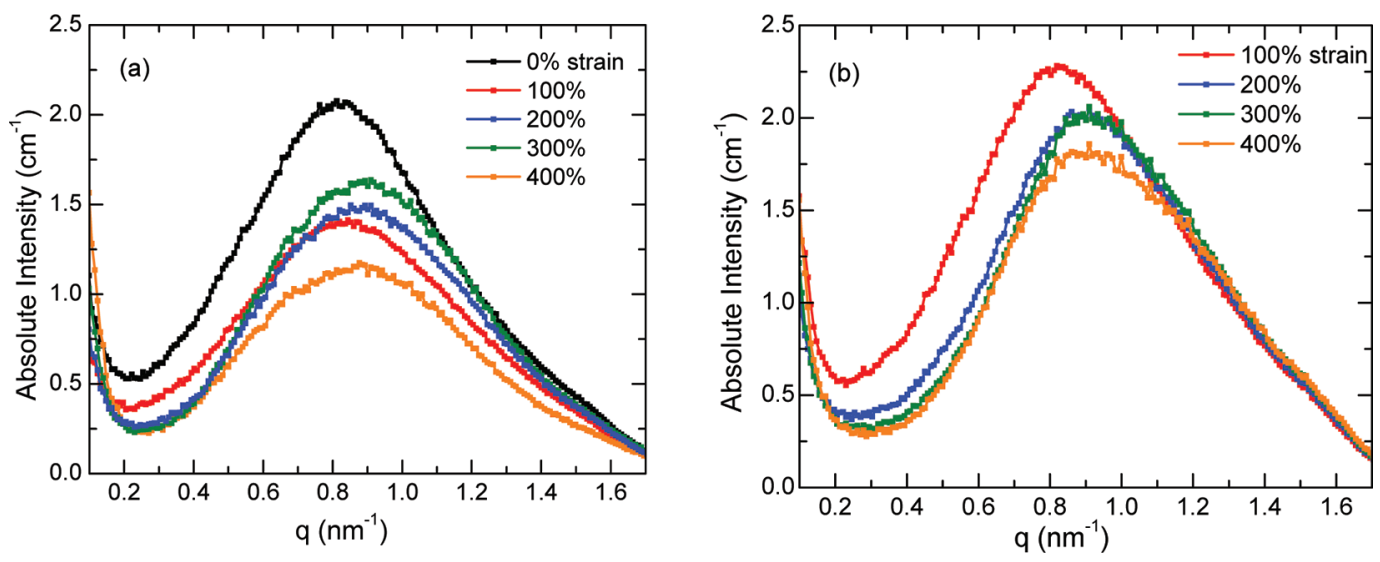

Figure 7. Background corrected absolute scattering intensity (azimuthally averaged) vs $q$ (a) under uniaxial extension and (b) relaxed after deformation.

degree of microphase separation (equal to zero for complete mixing and unity for phase-separation without intermixing). The hypothetical electron density variance for a two-phase system $\left(\overline{\Delta \eta_{c}^{2}}\right)$, assuming complete phase separation between hard and soft segments with sharp phase boundaries is given by ${ }^{28}$

$$
\overline{\Delta \eta_{c}^{2}}=\phi_{h s} \phi_{s s}\left(\eta_{h s}-\eta_{s s}\right)^{2}=\phi_{h s}\left(1-\phi_{h s}\right)\left(\eta_{h s}-\eta_{s s}\right)^{2}
$$

where $\phi_{h s}$ and $\phi_{s s}$ are the respective volume fractions of hard and soft segments in (hypothetical) completely phase-separated copolymers, and $\eta_{h s}$ and $\eta_{s s}$ are the corresponding electron densities. In our calculations of the completely demixed case, we assume that Isonate $143 \mathrm{~L}$, polyurea linkages, and phenyl rings from the soft segment end groups comprise the hard phase, and the PTMO and two carbonate groups of the soft segments are in the soft phase. $\overline{\Delta \eta_{c}^{2}}$ is calculated based on 
Table 1. Mean Interdomain Spacing, Experimental Electron Density Variances, Boundary Size, and Variance Ratios for Microphase Separation, Boundary, and Intermixing Contributions to the Scattering from Deformed and Relaxed Polyureas

\begin{tabular}{|c|c|c|c|c|c|c|c|c|c|}
\hline & $\begin{array}{c}d=2 \pi / \\
q_{\max }(\mathrm{nm})\end{array}$ & $\begin{array}{c}\overline{\Delta \eta^{2 \prime}} \times 1000 \\
\left(\mathrm{~mol} \mathrm{e}^{-} / \mathrm{cm}^{3}\right)^{2}\end{array}$ & $\begin{array}{l}\text { degree of microphase } \\
\text { separation } \overline{\Delta \eta^{21}} / \overline{\Delta \eta_{c}^{2}}\end{array}$ & $\begin{array}{l}\text { boundary } \\
\text { size }(\sigma, \mathrm{nm})\end{array}$ & $\begin{array}{l}\text { boundary } \\
\text { diffuseness } \\
\overline{\Delta \eta^{2 \prime \prime}} / \overline{\Delta \eta^{2 \prime}-1}\end{array}$ & $\begin{array}{l}\text { intermixing within } \\
\text { microphases } \\
\frac{\Delta \eta_{c}^{2}}{/ \Delta \eta^{2 \prime}}-1\end{array}$ & $\begin{array}{c}\text { phase separation } \\
(\%)\end{array}$ & $\begin{array}{c}\text { boundary } \\
(\%)\end{array}$ & $\begin{array}{l}\text { intermixing } \\
(\%)\end{array}$ \\
\hline $\begin{array}{l}\text { undeformed } \\
\text { polyurea }\end{array}$ & 7.4 & 2.11 & 0.38 & 0.54 & 0.74 & 0.52 & 38 & 36 & 26 \\
\hline \multicolumn{10}{|c|}{ Deformed } \\
\hline $100 \%$ strain & 7.4 & 1.44 & 0.26 & 0.54 & 0.94 & 1.00 & 26 & 36 & 38 \\
\hline $200 \%$ strain & 7.0 & 1.55 & 0.28 & 0.54 & 0.95 & 0.85 & 28 & 38 & 34 \\
\hline $300 \%$ strain & 7.0 & 1.63 & 0.29 & 0.53 & 0.96 & 0.74 & 29 & 40 & 31 \\
\hline $400 \%$ strain & 7.2 & 1.14 & 0.20 & 0.52 & 0.96 & 1.50 & 20 & 31 & 49 \\
\hline \multicolumn{10}{|c|}{ Relaxed } \\
\hline $100 \%$ strain & 7.7 & 2.30 & 0.41 & 0.55 & 0.94 & 0.25 & 41 & 46 & 12 \\
\hline $200 \%$ strain & 6.9 & 2.15 & 0.39 & 0.55 & 1.01 & 0.29 & 39 & 48 & 14 \\
\hline $300 \%$ strain & 6.9 & 2.16 & 0.39 & 0.55 & 1.06 & 0.25 & 39 & 50 & 12 \\
\hline $400 \%$ strain & 6.9 & 2.04 & 0.37 & 0.55 & 1.09 & 0.30 & 37 & 50 & 14 \\
\hline
\end{tabular}

$100 \%$ stoichoimetry of amine and isocyanate (4:1 weight ratio) using $144.5 \mathrm{~g}$ equiv weight for the isocyante and a functionality of Isonate $143 L=2.1$, as reported by the manufacturer. ${ }^{29}$ Since $\overline{\Delta \eta_{c}^{2}}$ is calculated using the chemical composition of the polyurea, the theoretical value is the same for all samples in this study; $\overline{\Delta \eta_{c}^{2}} \times 1000=5.57\left(\mathrm{~mol} \mathrm{e}-/ \mathrm{cm}^{3}\right)^{2}$.

Interface boundary diffuseness and segment intermixing can also be determined from the SAXS data as follows. With the assumption of a sigmoidal-gradient for the interface, the background corrected scattering intensity (in absolute units) is given by $^{20,30}$

$$
\begin{aligned}
I_{o b s}(q) & =I(q)-I_{b}(q) \\
& =I_{i d}(q) H^{2}(q) \\
& =I_{i d}(q) \exp \left(-\sigma^{2} q^{2}\right) \\
& =I_{i d}(q)\left(1-\sigma^{2} q^{2}\right)
\end{aligned}
$$

at large $q$, where $I_{i d}(q)$ is the ideal scattering intensity, $H(q)$ is the Fourier transform of a smoothing function characterizing the shape and size of the diffuse boundaries, and $\sigma$ is the diffuse boundary layer length. Because the ideal scattering intensity obeys Porod law's, ${ }^{30}$ eq 5 can be written as:

$$
I_{o b s}(q)=\frac{K}{q^{4}}\left(1-\sigma^{2} q^{2}\right)
$$

Therefore, for data in the form of $q^{4} I(q)$ vs $q^{2}, \sigma$ is obtained from the slope $\left(K \sigma^{2}\right)$ and $y$-intercept $(K)$ at larger $q$ (see Table 1$)$.

A second electron density variance $\left(\overline{\Delta \eta^{2 \prime \prime}}\right)$ can be calculated that removes the contribution to the scattering from diffuse boundaries. $^{28} \overline{\Delta \eta^{2 \prime \prime}}$ is defined as ${ }^{27}$

$$
\overline{\Delta \eta^{2 \prime \prime}}=c \int_{0}^{\infty} \frac{\left[\left(I(q)-I_{b}(q)\right] q^{2}\right.}{H^{2}(q)} \mathrm{d} q
$$

where $H(q)=\exp \left(\left(-\left(\sigma^{2} q^{2}\right) / 2\right)\right)$. The quantity $\left[\overline{\Delta \eta^{2 \prime \prime}} / \overline{\Delta \eta^{2 \prime}}-1\right]$ is a measure of the boundary diffuseness, and similarly $\left[\overline{\Delta \eta_{c}^{2}} /\right.$ $\left.\overline{\Delta \eta^{2 \prime}}-1\right]$ provides a measure of the influence of intermixed segments on overall phase separation.

Values of these three ratios are displayed in Table 1. The final three columns in the table describe the findings in a somewhat different fashion. For the undeformed polyurea,
$\overline{\Delta \eta^{2 \prime}} / \overline{\Delta \eta_{c}^{2}}=0.38$ (i.e., $38 \%$ phase separation). The relative contribution of the diffuse interface to the scattered intensity is $36 \%$. The remainder of the deviation from $\overline{\Delta \eta_{c}^{2}}, 26 \%$, we attribute to unlike segment mixing within the microphases.

As seen in Table 1, the mean spacing between hard domains is $7 \mathrm{~nm}$ for all samples, regardless of deformation or relaxation. The value of $\sigma(\sim 0.55 \mathrm{~nm})$ is also the same for all conditions; this quantity represents the standard deviation of the Gaussian function used in the sigmoidal-gradient model. This corresponds to $1.9 \mathrm{~nm}$ for the boundary layer thickness $\left((12)^{1 / 2} \sigma\right)$ in the easier to visualize but less physically realistic linear-gradient model. $^{30}$

With increasing strain, intersegment microphase separation is significantly reduced, so that the intermixing contribution to the scattering increases. The boundary contribution is the same within experimental uncertainty for the undeformed and strained materials, except for the case of $400 \%$ strain where the boundary contribution decreases somewhat. ${ }^{31}$ It is rather likely that hard domains consist of hard segments with little or no soft segments due to the strong interurea bidentate hydrogen bonding formed upon hard segment segregation. Therefore, the physical picture that emerges is hard domain orientation and disruption on straining polyurea to substantial levels, causing hard segments to become increasingly associated with the soft phase.

After relaxation for $24 \mathrm{~h}$, all previously strained samples exhibit a similar level of microphase separation as the undeformed sample, indicating that hard segments rearrange significantly during relaxation. In addition, relaxation leads to an increase in the diffuse boundary contribution to the scattering, and a corresponding lower degree of unlike segment mixing. However, as demonstrated by the 2D SAXS results, the hard domains after relaxation retain substantial orientation after their deformation by the applied strain.

Segmental Dynamics. There have been several investigations of the effect of mechanical strain on the segmental dynamics of polymers, using dynamic mechanical ${ }^{32-34}$ and broadband dielectric spectroscopies. ${ }^{35-39}$ In single phase elastomers, strain has been observed to have no effect, ${ }^{34,37}$ to speed up, ${ }^{32}$ or to slow down ${ }^{35,36}$ segmental relaxation, with even a nonmonotonic response to strain reported. ${ }^{33}$ These effects have been rationalized in terms of two competing effects: the decrease of configurational entropy due to chain orientation leading to longer relaxation times, and a small decrease of density (due to 
the small, but finite compressibility) tending to speed up the dynamics. However, these effects are generally small, if observed at all, corresponding to changes of the glass transition temperature of a few degrees or less. Thermoplastic elastomers, on the other hand, can show very large effects of strain on their dynamics. We recently studied the effects of strain on the dynamics of a polyurethane based on poly(tetramethylene oxide) soft segments and MDI/1,4-butanediol hard segments, which has similarities to the present polyurea material. The polyurethane selfassembles into a morphology consisting of quasi-spherical hard domains embedded in a soft matrix, ${ }^{40}$ compared to the highly elongated hard domains of the polyurea studied herein ${ }^{41}$ and some other polyureas. ${ }^{42}$ With increasing strain, the soft segment $\alpha$-relaxation of the polyurethane becomes significantly slower and weaker, with a broader distribution of relaxation times. ${ }^{43}$

In Figure 8 are shown dielectric spectra of dry and hydrated polyurea, both unstretched and at the maximum studied strain $(400 \%)$ at a representative temperature $(298 \mathrm{~K})$. In the tem-

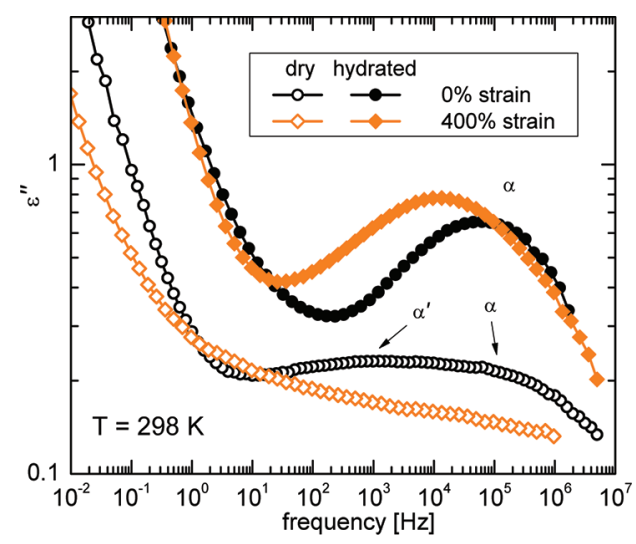

Figure 8. Dielectric loss spectra of dry (open symbols) and hydrated (filled symbols) polyurea at $298 \mathrm{~K}$, undeformed and at $400 \%$ strain.

perature range studied, the chain segments in the hard domains are immobile, so that the loss peaks are due to the segmental dynamics of the soft phase. The apparent increase in the loss at low frequencies is due to a combination of dc conductivity, interfacial polarization (i.e., mobile ions trapped at the interfaces between hard and soft domains), and possibly other relaxation modes such as local motion of the hard segments. ${ }^{44}$

In the unstretched, dry polyurea, a very broad double peak is observed. On the basis of the behavior when changing stoichiometry, temperature, or pressure, we previously attributed the lower-frequency component ( $\alpha^{\prime}$ process) to segmental relaxation of the soft phase in the interfacial regions between the soft and hard domains, while the primary, higher-frequency component was ascribed to segmental relaxation in the soft phase distant from interfaces. ${ }^{3}$ With increasing water content the two components combine into a single $\alpha$-peak. This process reflects the overall segmental dynamics of the soft phase (slightly plasticized compared to the dry polyurea) and the temperature for which $\tau_{\alpha}=100 \mathrm{~s}$ roughly corresponds to the calorimetric glass transition temperature. ${ }^{3}$

At high strains, the segmental relaxation of the dry polyurea moves to lower frequency, broadens, and exhibits much weaker intensity; the peak becomes obscured by the background loss and cannot be further analyzed. In the hydrated sample, the segmental relaxation moves to lower frequencies but remains prominent even at high strains. Therefore, in the following, we show dielectric data only for hydrated samples under strain.

Figure 9 shows dielectric loss spectra of hydrated polyurea at $273 \mathrm{~K}$ at different static strains. With increasing extension, the

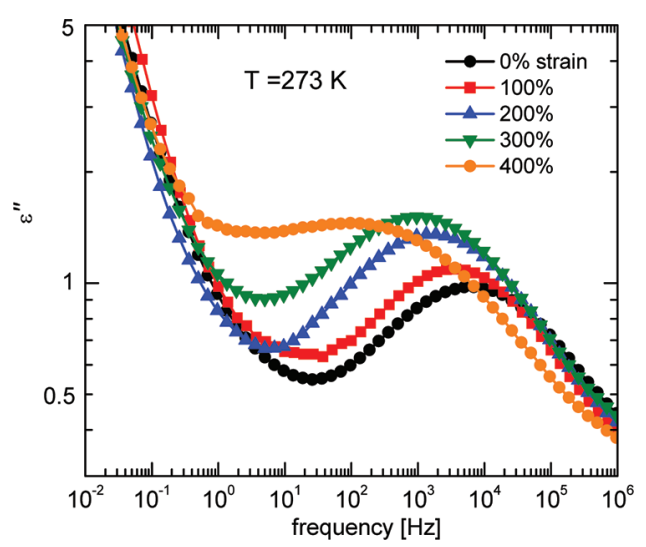

Figure 9. Dielectric spectra of hydrated polyurea at $273 \mathrm{~K}$ under uniaxial deformation. With increasing strain, the segmental relaxation peak moves to lower frequencies and broadens.

peak moves systematically toward lower frequencies, and becomes more intense and broader. The increased amplitude of the peak in the hydrated sample is opposite to the behavior observed in the dry samples. However, it should not be attributed to increased mobility, but rather to the increase in equilibrium water content with increased strain (from $2.4 \%$ in the undeformed sample to $3.2 \%$ at $400 \%$ strain), since the intensity of the soft segment $\alpha$-relaxation increases very strongly with increasing water content. ${ }^{3}$ This increased hydration presumably arises due to the breakup of hard segments, effected by $\mathrm{H}$-bond breakage between the urea segments, thus exposing additional, highly polar, hydration sites.

We fit the dielectric spectra to a sum of a Havriliak-Negami function ${ }^{45}$ for the segmental process and a power law for the low-frequency loss:

$$
\varepsilon^{*}(f)=\varepsilon_{\infty}+\frac{\Delta \varepsilon}{\left[1+\left(i f / f_{H N}\right)^{a}\right]^{b}}+\frac{\sigma}{i f^{n}}
$$

where $\varepsilon_{\infty}$ is the infinite frequency dielectric constant, $\Delta \varepsilon$ the intensity of the relaxation (contribution to the static dielectric constant), $a$ and $b$ are shape parameters, with $a, b>0$ and $a<1$, $a b \leq 1, \sigma$ and $n$ are temperature-dependent constants, and $f_{H N}$ is related to the frequency of maximum loss $f_{\mathrm{maz}}=1 /\left(2 \pi \tau_{a}\right)$ by

$$
f_{\text {max }}=f_{H N}\left(\frac{\sin (\pi a /(2+2 b))}{\sin (\pi a b /(2+2 b))}\right)^{1 / a}
$$

Segmental relaxation frequencies are plotted against temperature in Figure 10. The $\alpha$ relaxation becomes slower with increasing strain over the entire frequency range; however, the effect is stronger close to $T_{\mathrm{g}}$, as the curves converge at high temperatures. The relaxation times are well described over the measured range by the Vogel-Fulcher function. ${ }^{46-48}$

$$
f_{\max }=f_{0} \exp \left(-\frac{B}{T-T_{0}}\right)
$$

where $T_{0}$ (Vogel temperature), $f_{0}$, and $B$ are constants. The parameters of eq 8 are listed in Table 2, along with the dynamic 


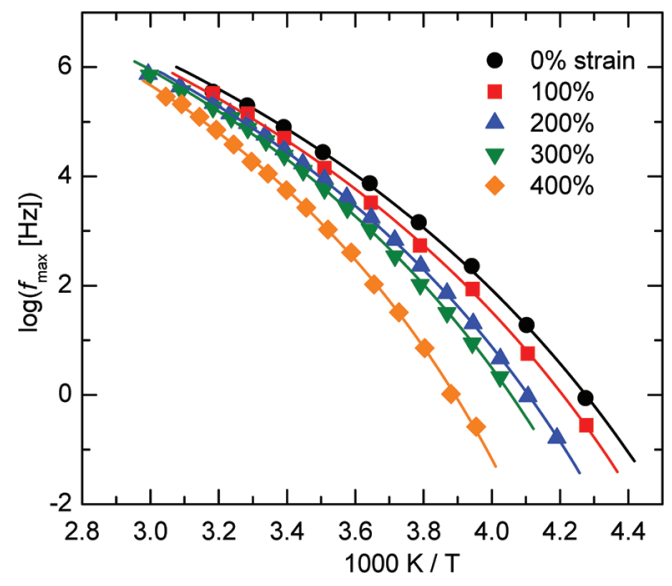

Figure 10. Segmental relaxation times of polyurea under uniaxial deformation. Lines are fits to the Vogel-Fulcher equation.

glass transition temperature (defined as the temperature at which $\tau_{a}=100 \mathrm{~s}$ ). Strain has no effect on $f_{0}$, but significantly

Table 2. Parameters of Equation 8, Glass Transition Temperatures (Defined as the Temperature Where $\tau=100 \mathrm{~s}$ ) and Fragilities for Hydrated Deformed Polyurea

\begin{tabular}{lcrrrr}
\multicolumn{1}{c}{ strain } & $\log \left(f_{0} / \mathrm{Hz}\right)$ & $B[\mathrm{~K}]$ & $T_{0}[\mathrm{~K}]$ & $T_{\mathrm{g}}[\mathrm{K}]$ & $m$ \\
\hline undeformed polyurea & 10.1 & 1506 & 71.2 & 218.6 & 64 \\
$100 \%$ strain & 10.2 & 1603 & 169.4 & 222.0 & 65 \\
$200 \%$ strain & 10.4 & 1638 & 175.5 & 228.3 & 69 \\
$300 \%$ strain & 10.7 & 1754 & 175.9 & 231.5 & 71 \\
$400 \%$ strain & 11.1 & 1894 & 183.2 & 242.4 & 76
\end{tabular}

increases $T_{\mathrm{g}}$. Given the increased water content at higher strain, we might expect the material to be more plasticized at larger strains (although the effect would be minor); therefore, if compared at equivalent water content, the increase in $T_{\mathrm{g}}$ with strain may in fact be even larger. At $400 \% T_{\mathrm{g}}$ is more than $20 \mathrm{~K}$ higher than for the undeformed sample. Also listed in Table 2 and plotted in Figure 11 is the fragility, defined as ${ }^{49}$

$$
m=\left.\frac{\mathrm{d}(\log \tau)}{\mathrm{d}\left(T_{0} / T\right)}\right|_{T=T_{\mathrm{g}}}
$$

The fragility quantifies the non-Arrhenius character of the temperature dependence of $\tau_{\alpha}$; a stronger deviation from Arrhenius behavior corresponds to a more fragile system. With increasing strain, relaxation times become more sensitive to temperature, and the fragility increases. A similar strain dependence of $T_{\mathrm{g}}$ and $m$ was observed for uniaxially oriented polyurethane. ${ }^{43^{8}}$ The increased fragility with strain fits the general, albeit weak, correlation of fragility with $T_{\mathrm{g}}{ }^{50}$ Fragility has been demonstrated to increase with increasing chemical cross-linking, ${ }^{51,52}$ as well as intermolecular hydrogen bonding, ${ }^{53}$ so the increase observed herein is consistent with greater constraints imposed on the soft segment motion due to more intimate association with hard segments. The latter contains some trifunctional units (chemical cross-links), as well as urea groups capable of forming hydrogen bonds with the ether oxygen atoms on the soft segments.

Figure 12 shows the full width at half-maximum of the segmental relaxation, quantifying the breadth of the distribution of relaxation times. The segmental process becomes broader

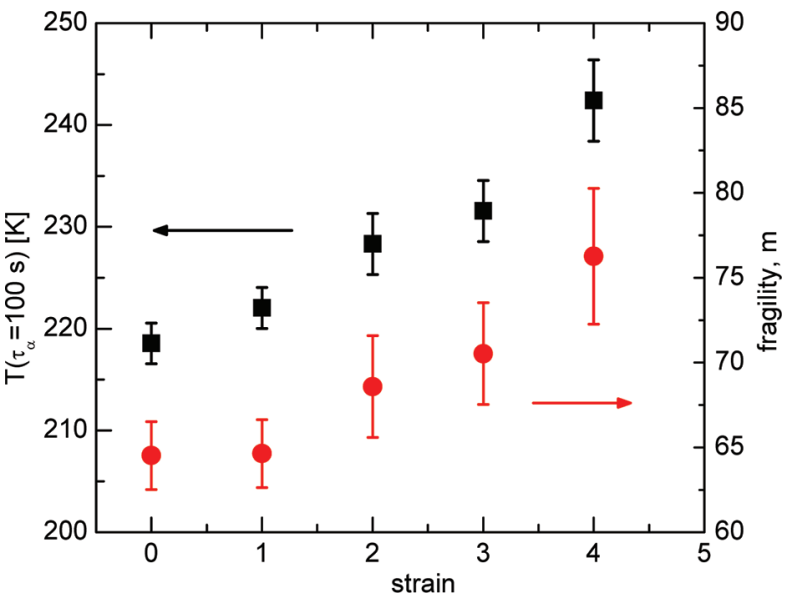

Figure 11. Glass transition temperature and fragility of uniaxially deformed polyurea (defined as the temperature where the segmental relaxation time is $100 \mathrm{~s}$ ) as a function of applied strain.

with decreasing temperature, as is usual for glass-forming systems. At constant temperature, the peak broadens with

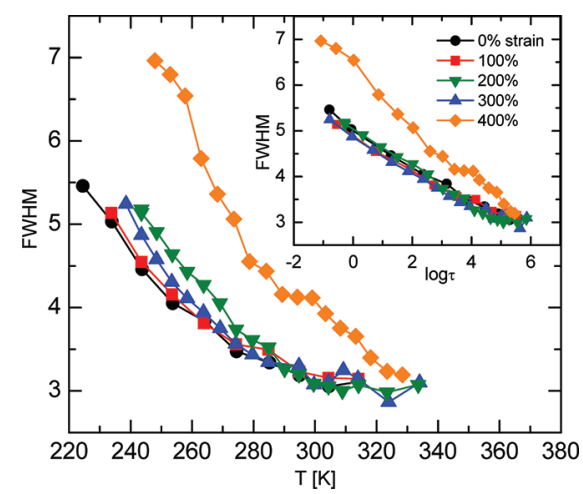

Figure 12. Full width at half-maximum of the segmental relaxation of uniaxially deformed polyurea as a function of temperature (also plotted as a function of segmental relaxation time in the inset).

increasing strain. However, plotting the peak width against $\tau_{\alpha}$ collapses the curves except for the highest (400\%) strain. Therefore, the broadening at constant temperature at strains up to $300 \%$ is due only to the strain-induced shift toward lower frequencies, whereas at $400 \%$ strain the distribution of relaxation times actually broadens significantly. The breadth of the distribution of relaxation times reflects heterogeneity; both the dynamic heterogeneity inherent to the supercooled dynamics of liquids and polymers, and an additional contribution from the structural heterogeneity of the local chain environments; i.e., regions with different composition have different restrictions imposed on their motions by neighboring hard domains. With increasing strain, despite mixing of hard segments into the soft phase, this structural heterogeneity does not appear to change substantially below $400 \%$ strain.

\section{SUMMARY}

Previously it was shown that while high strain rates induce primarily an elastic response, with only modest permanent changes in morphology and sample dimensions, the situation is quite different for slow extension of elastomeric polyurea. ${ }^{1}$ In this work, we examined the detailed dependence of the latter on the magnitude of the deformation. In the neo-Hookean 
region there is no permanent effect of strain, whereas deformations beyond the yield stress gives rise to unrecovered strain and orientation of the domains, along with disruption of the hard domains with consequent reduction in the degree of phase segregation. These morphological changes, in turn, cause significant slowing down of the segmental dynamics and broadening of its distribution of relaxation times and a large increase of apparent activation energy (fragility). The extent of these changes in the material varies continuously over the course of the deformation.

Polyurea is unrivaled in terms of the complexity of its mechanical behavior; nevertheless, extensive efforts have been made to model polyureas, particularly for impact applications. ${ }^{15-18}$ As is generally the case for elastomers, the modeling efforts usually rely on assumptions of separability between strain and strain rate effects. Certainly this assumption breaks down at strain rates sufficient for the rubber to undergo a viscoelastic transition to the glassy state. ${ }^{7}$ However, the present results demonstrate that even at very slow rates, the material per se and thus its mechanical response vary continuously over the course of deformation. Thus, realistic modeling of polyurea requires consideration of the instantaneous state of the morphology, which is convoluted with other factors such as strain nonlinearity, viscoelasticity, as well as other factors such as the effect of hydrostatic pressure and absorbed moisture. ${ }^{2,3}$

\section{AUTHOR INFORMATION}

\section{Corresponding Author}

*E-mail: (J.R.) runt@matse.psu.edu; (C.M.R.) mike.roland@ nrl.navy.mil.

\section{Notes}

The authors declare no competing financial interest.

\section{ACKNOWLEDGMENTS}

T.C. and J.R. gratefully acknowledge the National Science Foundation, Polymers Program, under Grant DMR-0907139 and the Office of Naval Research Contract Number 4036-CUONR-1125 for their support of this research. D.F. acknowledges a postdoctoral fellowship from the National Research Council. The work at NRL was supported by the Office of Naval Research, Code 331.

\section{REFERENCES}

(1) Pathak, J. A.; Twigg, J. N.; Nugent, K. E.; Ho, D. L.; Lin, E. K.; Mott, P. H.; Robertson, C. G.; Vukmir, M. K.; Epps, T. H. III; Roland, C. M. Macromolecules 2008, 41, 7543-7548.

(2) Roland, C. M.; Casalini, R. Polymer 2007, 48, 5747-5752.

(3) Fragiadakis, D.; Gamache, R.; Bogoslovov, R. B.; Roland, C. M. Polymer 2010, 51, 178-184.

(4) Roland, C. M.; Casalini, R. Polymer 2007, 48, 5747-5752.

(5) Shim, J.; Mohr, D. Int. J. Impact Eng. 2009, 36, 1116-1127.

(6) Yi, J.; Boyce, M. C.; Lee, G. F.; Balizer, E. Polymer 2006, 47, 319329.

(7) Bogoslovov, R. B.; Roland, M. J. Appl. Phys. 2007, 102, 063531.

(8) Sarva, S. S.; Deschanel, S.; Boyce, M. C.; Chen, W. Polymer 2007, $48,2208-2213$.

(9) Xue, L.; Mock, W. Jr.; Belytschko, T. Mech. Mater. 2010, 42, 981-1003.

(10) Bogoslovov, R. B.; Roland, C. M.; Gamache, R. M. Appl. Phys. Lett. 2007, 90, No. in press.

(11) Tekalur, S. A.; Shukla, A.; Shivakumar, K. Compos. Struct. 2008, 84, 271-281.

(12) Davidson, J. S.; Fisher, J. W.; Hammons, M. I.; Porter, J. R.; Dinan, R. J. J. Struct. Eng.-ASCE 2005, 131, 1194-1205.
(13) Jiao, T.; Clifton, R. J.; Grunschel, S. E. High strain rate response of an elastomer. In Shock Compression of Condensed Matter-2005, Parts 1 and 2; 2006; Vol. 845, pp 809-812.

(14) Roland, C. M.; Fragiadakis, D.; Gamache, R. M. Compos. Struct. 2010, 92, 1059-1064.

(15) Shim, J.; Mohr, D. Int. J. Plast. 2011, 27, 868-886.

(16) Amirkhizi, A. V.; Isaacs, J.; McGee, J.; Nemat-Nasser, S. Philos. Mag. 2006, 86, 5847-5866.

(17) Grujicic, M.; Pandurangan, B.; King, A. E.; Runt, J.; Tarter, J.; Dillon, G. J. Mater. Sci. 2011, 46, 1767-1779.

(18) Rinaldi, R. G.; Boyce, M. C.; Weigand, S. J.; Londono, D. J.; Guise, M. W. J. Polym. Sci., Part B: Polym. Phys. 2011, 49, 1660-1671.

(19) Russell, T. P.; Lin, J. S.; Spooner, S.; Wignall, G. D. J. Appl. Crystallogr. 1988, 21, 629-638.

(20) Roe, R. J. Methods of X-ray and Neutron Scattering in Polymer Science; Oxford University Press: New York, 2000.

(21) Yeh, F.; Hsiao, B. S.; Sauer, B. B.; Michel, S.; Siesler, H. W. Macromolecules 2003, 36, 1940-1954.

(22) Bonart, R.; Morbitze., L.; Hentze, G. J. Macromol. Sci.-Phys. 1969, B3, 337.

(23) Bonart, R. J. Macromol. Sci., Part B 1968, 2, 115-38.

(24) Desper, C. R.; Schneider, N. S.; Jasinski, J. P.; Lin, J. S. Macromolecules 1985, 18, 2755-2761.

(25) Waletzko, R. S.; Korley, L. T. J.; Pate, B. D.; Thomas, E. L.; Hammond, P. T. Macromolecules 2009, 42, 2041-2053.

(26) Ran, S. F.; Fang, D. F.; Sics, I.; Toki, S.; Hsiao, B. S.; Chu, B. Rev. Sci. Instrum. 2003, 74, 3087-3092.

(27) Garrett, J. T.; Runt, J.; Lin, J. S. Macromolecules 2000, 33, 63536359.

(28) Bonart, R.; Muller, E. H. J. Macromol. Sci-Phys. 1974, B10, 177-189.

(29) Polyols \& Isocyanates for Coating, Adhesive, Sealant and Elastomer Applications in North America, Dow Chemical, 2009, http://www.dow.com/polyurethane/na/pdfs/109-01823.pdf

(30) Koberstein, J. T.; Morra, B.; Stein, R. S. J. Appl. Crystallogr. 1980, 13, 34-45.

(31) Sonnenschein, M. F.; Prange, R.; Schrock, A. K. Polymer 2007, $48,616-623$.

(32) Diaz-Calleja, R.; Riande, E.; Guzman, J. Polymer 1987, 28, 2190-2194.

(33) Riande, E.; Guzman, J.; Llorente, M. A. J. Polym. Sci., Part B: Polym. Phys. 1983, 21, 2473-2482.

(34) Munch, E.; Pelletier, J. M.; Sixou, B.; Vigier, G. Polymer 2006, 47, 3477-3485.

(35) Naoki, M.; Nakajima, K.; Nose, T.; Hata, T. Polym. J. 1974, 6, 283-294.

(36) Nakajima, K.; Naoki, M.; Nose, T. Polym. J. 1978, 10, 307-314.

(37) Adachi, H.; Adachi, K.; Kotaka, T. Polym. J. 1980, 12, 329-334.

(38) Diaz-Calleja, R.; Riande, E.; Guzman, J. J. Polym. Sci., Part B: Polym. Phys. 1990, 28, 1551-1563.

(39) Hernandez, M.; Lopez-Manchado, M. A.; Sanz, A.; Nogales, A.; Ezquerra, T. A. Macromolecules 2011, 44, 6574-6580.

(40) Hernandez, R.; Weksler, J.; Padsalgikar, A.; Choi, T.; Angelo, E.; Lin, J. S.; Xu, L.-C.; Siedlecki, C. A.; Runt, J. Macromolecules 2008, 41, 9767-9776.

(41) Castagna, A.; Dillon, G.; Runt, J. Manuscript in preparation.

(42) Das, S.; Yilgor, I.; Yilgor, E.; Wilkes, G. L. Polymer 2008, 49, 174-179.

(43) Lee, H.; Fragiadakis, D.; Martin, D.; Milne, A.; Milne, J.; Runt, J. Macromolecules 2010, 43, 3125-3127.

(44) Castagna, A.; Fragiadakis, D.; Lee, H.-K.; Choi, T.; Runt, J. Macromolecules 2011, 44, 7831-7836.

(45) Havriliak., S.; Negami, S. J. Polym. Sci., Part C: Polym. Symp. 1966, 99.

(46) Fulcher, G. S. J. Am. Ceram. Soc. 1925, 8, 339-355.

(47) Tammann, G.; Hesse, W. Z. Anorg. Allgem. Chem. 1926, 156.

(48) Vogel, H. Phys. Z. 1921, 22, 645-646.

(49) Bohmer, R; Angell, C. A. Phys. Rev. B 1992, 45, 10091-10094. 
(50) Qin, Q.; McKenna, G. B. J. Non-Cryst. Solids 2006, 352, 29772985.

(51) Kramarenko, V. Y.; Ezquerra, T. A.; Sics, I.; Balta-Calleja, F. J.; Privalko, V. P. J. Chem. Phys. 2000, 113, 447-452.

(52) Roland, C. M. Macromolecules 1994, 27, 4242-4247.

(53) Zhang, S. H.; Painter, P. C.; Runt, J. Macromolecules 2002, 35, 9403-9413. 